\title{
Lattice QCD at finite temperature and density in the phase-quenched approximation
}

\author{
J. B. Kogut* \\ Department of Energy, Division of High Energy Physics, Washington, DC 20585, USA \\ and \\ Dept. of Physics - TQHN, Univ. of Maryland, \\ 82 Regents Dr., College Park, MD 20742, USA \\ D. K. Sinclain $\dagger^{\dagger}$ \\ HEP Division, Argonne National Laboratory, \\ 9700 South Cass Avenue, Argonne, IL 60439, USA
}

\begin{abstract}
QCD at a finite quark-number chemical potential $\mu$ has a complex fermion determinant, which precludes its study by standard lattice QCD simulations. We therefore simulate lattice QCD at finite $\mu$ in the phase-quenched approximation, replacing the fermion determinant with its magnitude. (The phase-quenched approximation can be considered as simulating at finite isospin chemical potential $2 \mu$ for $N_{f} / 2 u$-type and $N_{f} / 2 d$-type quark flavours.) These simulations are used to study the finite temperature transition for small $\mu$, where there is some evidence that the position (and possibly the nature) of this transition are unchanged by this approximation. We look for the expected critical endpoint for 3-flavour QCD. Here, it had been argued that the critical point at zero $\mu$ would become the critical endpoint at small $\mu$, for quark masses just above the critical mass. Our simulations indicate that this does not happen, and there is no such critical endpoint for small $\mu$. We discuss how we might adapt techniques used for imaginary $\mu$ to improve the signal/noise ratio and strengthen our conclusions, using results from relatively low statistics studies.
\end{abstract}

\footnotetext{
*Supported in part by NSF grant NSF PHY03-04252.

$\dagger$ This work was supported in part by the U.S. Department of Energy, Division of High Energy Physics, Contract DE-AC02-06CH11357.
} 


\section{INTRODUCTION}

Relativistic heavy-ion colliders allow one to study hadronic and nuclear matter at high temperatures where it undergoes a transition to a quark-gluon plasma. While the highest energy colliders (RHIC and the forthcoming heavy-ion program at the LHC) study only the very low density regime where the baryon-number density is too small to have much effect on the thermodynamics, lower energy relativistic heavy-ion colliders can probe the region where baryon-number density is appreciable.

For physical $u, d$ and $s$ quark masses, the finite temperature transition at zero baryonnumber density is predicted to be a rapid crossover rather than a true phase transition [1, 2, 3]. It is expected that, at high enough baryon-number densities, this transition will become first order. The point at which the change from a crossover to a first-order transition occurs would be a critical point, expected to be in the universality class of the 3-dimensional Ising model. This critical point is referred to as a critical endpoint, and is expected to be the most interesting feature of this intermediate density regime of the QCD phase diagram.

While finite temperature QCD is straightforward (but tedious) to simulate on the lattice, QCD at a finite quark-number chemical potential $\mu$ has proved intractable. The reason is that at finite $\mu$ the fermion determinant becomes complex, with a real part having an indefinite sign. Since all the standard lattice QCD simulation methods rely on importance sampling, they fail for such systems.

In the region of small $\mu$, close to the finite-temperature phase transition, methods have been developed to circumvent this sign problem. These methods fall into several classes. One such method involves simulating lattice QCD at a carefully selected set of parameters where no such sign problem exists and using the ratios of determinants to reweight to the region

of interest [4]. Such multiparameter reweighting only works provided there is significant overlap between those configurations which are important for the chosen set of parameters, and those which are important for the original set of parameters. A second class of methods are those which rely on analyticity in $\mu$ or related parameters. These include series expansion methods [5, 6], which expand the Boltzmann weight and the observables as power series in $\mu$, calculating the coefficients in simulations at zero $\mu$. Since the higher order coefficients require the calculation of higher order fluctuation quantities, this ultimately limits their utility. Other analyticity methods involve simulating in a domain of parameters such as at 
imaginary $\mu$, where there is no sign problem, and analytically continuing the results to the desired domain (in this case, real $\mu$ ), typically by fitting the results to a power series [7, 8]. There exist variants where different parameters are used for the analytic continuation such as [9]. Another way of avoiding the sign problem is to use canonical methods [10, 11, 12]. Here the sign problem is encountered in Fourier transforming to obtain the canonical ensembles at fixed quark number.

We have adopted the alternative approach of ignoring the phase of the determinant and replacing the determinant by its magnitude. This can be thought of as simulating QCD with $N_{f} / 2 u$ type quarks and $N_{f} / 2 d$ type quarks, with a chemical potential $\mu_{I}=2 \mu$ for isospin $\left(I_{3}\right)$. ( $N_{f}$ is the number of quark flavours.) For low temperatures, there is a critical point $\mu_{I}=\mu_{c}$ above which the system enters a superfluid phase, with a charged pion condensate which breaks $I_{3}$ symmetry spontaneously [13, 14, 15]. At zero temperature $\mu_{c}=m_{\pi}$. Since this phase does not exist for QCD at finite $\mu$, the phase-quenched approximation breaks down at the boundary of this superfluid domain, if not before.

The Taylor series calculations of the Bielefeld-Swansea collaboration [5, 16] showed evidence that the $\mu$ dependence of the transition temperature $T_{c}$ for full 2-flavour QCD was similar if not identical to that of the phase-quenched approximation (finite isospin chemical potential), at small $\mu$. The $\mu$ dependence of this transition for full 2-flavour QCD, obtained from the imaginary quark-number chemical potential simulations of de Forcrand and Philipsen, [7] was consistent with being identical that observed in our direct simulation of the phase-quenched theory [17]. A random matrix model of 2-flavour QCD at finite temperature and chemical potentials also predicts that the dependence of $T_{c}$ on quark-number and isospin chemical potentials should be identical for $\mu<m_{\pi} / 2$ [18]. In addition Nambu-JonaLasinio models for QCD have transition temperatures which exhibit the same dependence on quark-number and isospin chemical potentials for $\mu<m_{\pi} / 2[19,20]$. This strongly suggests that the $\mu$ dependence of $T_{c}$ is the same for phase-quenched and full QCD for small $\mu$. We shall indicate later that the simulations discussed in this paper are consistent with this assumption. There is, however, one lattice result which contradicts this assumption. The 3-flavour calculations of the Bielefeld-Swansea collaboration indicate that while the $T_{c}$ dependence on quark-number and isospin chemical potentials are consistent at larger quark masses, they are not at small quark masses [21, 22]. However, because of large statistical errors, the observed difference in slopes was less than two standard deviations. In addition, 
these simulations were performed using the $\mathrm{R}$ algorithm, which could potentially introduce larger than expected updating errors, due to the discretization of molecular-dynamics 'time', in the fluctuation quantities used to obtain these results.

For 3-flavour QCD at zero chemical potentials, the finite temperature transition is first order at small quark mass $m$. For larger $m$ the transition softens to a crossover with no phase transition. At $m=m_{c}$, where the nature of the transition changes, the finite-temperature transition is a critical point in the universality class of the 3-dimensional Ising model [1]. Similar behaviour is seen for $2+1$-flavour QCD, and for the physical $u, d$ and $s$ quark masses, the transition is predicted to be a crossover [2, 3]. It has been suggested that $m_{c}$ would increase with increasing $\mu$, becoming the critical endpoint. If so it should be possible to tune this endpoint to be as close to $\mu=0$ as desired by choosing $m$ just above $m_{c}$.

Hence we simulate 3-flavour lattice QCD at $\mu_{I}<m_{\pi}$, for several masses close to $m_{c}$, and determine the nature of the finite-temperature phase transition using fourth-order Binder cumulants for the chiral condensate. For these studies we use simulations on $8^{3} \times 4,12^{3} \times 4$ and $16^{3} \times 4$ lattices. Our simulations indicate that there is no critical endpoint for $m>m_{c}(0)$, and $m_{c}\left(\mu_{I}\right)$ actually decreases (slowly) with increasing $\mu$. Preliminary results from these simulations have been reported at various conferences, the most recent being Lattice2007 [23]. This absence of the expected critical endpoint at small $\mu\left(\mu_{I}\right)$ has been observed by de Forcrand and Philipsen using analytic continuation from imaginary $\mu$ [2]. Our simulations use the exact RHMC algorithm [24], since in the inexact hybrid molecular-dynamics used in earlier simulations, the Binder cumulant had such strong $d t^{2}$ dependence as to lead to incorrect conclusions as to the nature of the transition [25].

The relatively weak dependence of the Binder cumulant on $\mu_{I}^{2}$ and the statistical errors in determining it, even in high statistics runs, make it difficult to determine the sign of the slope $d B_{4} / d \mu_{I}^{2}$ and hence $d m_{c} / d \mu_{I}^{2}$ with certainty. Similar difficulties occurred with the methods of de Forcrand and Philipsen, but they were able to calculate the slope directly with much higher precision, using reweighting methods [26]. We have performed studies which indicate that similar methods should work for the phase-quenched simulations. However, on the larger lattices we use, it is unclear whether these methods will be significantly more efficient than simply increasing statistics. As yet, we have insufficient statistics to achieve results for the slope of the Binder cumulant. However, we are already able to determine the slope of $\beta_{c}$. 
Section 2 describes phase-quenched lattice QCD. In section 3 we describe our simulations and results. Exploratory studies of reweighting techniques are described in section 4 . Section 5 is devoted to discussions and conclusions.

\section{PHASE-QUENCHED LATTICE QCD}

Phase-quenched lattice QCD with eight staggered quark flavours (or two staggered quark fields, each with four 'tastes') has the fermion action

$$
S_{f}=\sum_{\text {sites }}\left[\bar{\chi}\left[\not D\left(\frac{1}{2} \tau_{3} \mu_{I}\right)+m\right] \chi\right.
$$

where $\not D\left(\frac{1}{2} \tau_{3} \mu_{I}\right)$ is the standard staggered quark transcription of $\not D$ with the links in the $+t$ direction multiplied by $\exp \left(\frac{1}{2} \tau_{3} \mu_{I}\right)$ and those in the $-t$ direction multiplied by $\exp \left(-\frac{1}{2} \tau_{3} \mu_{I}\right)$. Since we are performing simulations outside of the superfluid phase, the explicit symmetrybreaking interaction of our earlier studies is unnecessary.

To simulate $N_{f}$ flavours using the RHMC algorithm, this is replaced by the pseudofermion action

$$
S_{p f}=p_{\psi}^{\dagger} \mathcal{M}^{-N_{f} / 8} p_{\psi}
$$

where $p_{\psi}$ are the momenta conjugate to the pseudo-fermion field $\psi[36]$. Here,

$$
\mathcal{M}=\left[\not D\left(\frac{1}{2} \mu_{I}\right)+m\right]^{\dagger}\left[\not D\left(\frac{1}{2} \mu_{I}\right)+m\right]
$$

In the RHMC algorithm $\mathcal{M}^{-N_{f} / 8}$ (and $\mathcal{M}^{ \pm N_{f} / 16}$ ) are replaced by rational approximations,

using a speculative lower bound [25]. It is interesting to note that these rational approximations provide similar infrared protection to what a symmetry-breaking interaction would give.

For 8 flavours, and indeed for any even number of flavours, $\mu_{I}$ has the interpretation of an isospin chemical potential, for a theory with $N_{f} / 2 u$-type quarks and $N_{f} / 2 d$-type quarks. Since we are interested in this phase-quenched theory as an approximation to QCD with a quark-number chemical potential $\mu=\mu_{I} / 2$, we are free to choose any integral $N_{f}$. In fact we shall work with $N_{f}=3$.

As we have shown in earlier work, the Binder cumulant which is used to extract the nature of the finite-temperature transition is very sensitive to the updating increment $d t$ 
in the older, inexact, hybrid molecular-dynamics $(\mathrm{R})$ algorithm [25]. This is the principal reason that we have switched to the RHMC algorithm.

As mentioned in the introduction, such theories are known to undergo a phase transition to a superfluid phase with a charged pion condensate and orthogonal charged pion excitations which are true Goldstone bosons at low temperatures, as $\mu_{I}$ is increased. At zero temperature this transition occurs at $\mu_{I}=\mu_{c}=m_{\pi}$. At high enough temperatures the system should be in the quark-gluon phase for all $\mu_{I}$, and no such transition is expected.

\section{SIMULATIONS AND RESULTS}

We perform simulations of 3-flavour lattice QCD at finite $\mu_{I}$ and temperature on $8^{3} \times 4$, $12^{3} \times 4$, and $16^{3} \times 4$ lattices. We use rational approximations to $\mathcal{M}^{-3 / 8}$ and $\mathcal{M}^{ \pm 3 / 16}$ in these RHMC simulations which are valid provided the spectrum of $\mathcal{M}$ is in the range $[1 \times$ $\left.10^{-4}, 25\right]$. (2 runs were made using smaller speculative lower bounds for testing purposes.) We performed runs with quark masses $m=0.02, m=0.025, m=0.03$ and $m=0.035$ on $8^{3} \times 4$ and $12^{3} \times 4$ lattices. At the lowest mass, we only ran simulations for $\mu_{I}=0$. For the other 3 masses we ran simulations at $\mu_{I}=0, \mu_{I}=0.2$ and $\mu_{I}=0.3$. In addition, we ran simulations on $16^{3} \times 4$ lattices at $m=0.03$ at all $3 \mu_{I}$ s and at $m=0.025$ with $\mu_{I}=0$. The masses are chosen such that the lower 2 masses lie below $m_{c}$ and the higher 2 masses lie above $m_{c}$. The choice of $\mu_{I}$ values is to cover the region $0 \leq \mu_{I}<m_{\pi}$, where $m_{\pi}$ is estimated to lie in the range $0.4 \lesssim m_{\pi}<0.5$ for $0.025 \leq m \leq 0.035$. (This comes from measurements of $m_{\pi}$ for $m=0.03, \mu_{I}=0$ at $\beta=5.10$ and $\beta=5.15$, which bracket the region of interest, on $8^{3} \times 16$ and $12^{3} \times 24$ lattices. Estimates for other $m$ values were made using PCAC.)

For our $12^{3} \times 4$ simulations, where we have the highest statistics, we have run for 300,000 length-1 trajectories for each of 4 (or more) $\beta$ values close enough to the transition to access this transition using Ferrenberg-Swendsen reweighting in $\beta$, at each $\left(m, \mu_{I}\right)$. For the $8^{3} \times 4$ and $16^{3} \times 4$ simulations we have performed 300,000 trajectory runs at each of $2 \beta$ s at each $\left(m, \mu_{I}\right)$. We have made 5 independent stochastic estimates of the chiral condensate $\bar{\psi} \psi$ and the isospin density $j_{0}^{3}=\partial S_{f} / \partial \mu_{I}$ after each trajectory, to enable us to make unbiased estimates of the susceptibilities and Binder cumulants.

For any observable $X$, the susceptibility $\chi_{X}$ is defined by

$$
\chi_{X}=\frac{V}{T}\left\langle\bar{X}^{2}-\langle\bar{X}\rangle^{2}\right\rangle
$$


where $V$ is the spatial volume and $T=1 / N_{t}$ is the temperature. The overlining of $X$ indicates that these are lattice averaged quantities. The fourth-order Binder cumulant for $X$ is defined by

$$
B_{4}=\frac{\left\langle(\bar{X}-\langle\bar{X}\rangle)^{4}\right\rangle}{\left\langle(\bar{X}-\langle\bar{X}\rangle)^{2}\right\rangle^{2}}
$$

[27]. These quantities are measured at the value $\beta=\beta_{0}$ of the simulation and extrapolated to nearby $\beta$ s, by Ferrenberg-Swendsen reweighting:

$$
\langle X\rangle_{\beta}=\frac{\left\langle\exp \left[-6(V / T)\left(\beta-\beta_{0}\right) S_{\square}\right] X\right\rangle_{\beta_{0}}}{\left\langle\exp \left[-6(V / T)\left(\beta-\beta_{0}\right) S_{\square}\right]\right\rangle_{\beta_{0}}}
$$

[28] where

$$
S_{\square}=1-\frac{1}{3} \operatorname{Re} \operatorname{Tr}_{\square} U U U U
$$

appropriately averaged over the lattice and over plaquette orientations. The position of the transition, $\beta_{c}$, can be estimated as that of the peak of the susceptibilities, or the minimum of the Binder cumulants. We have noticed that the measured $\beta_{c}$ s from the susceptibilities of various observables and from the Binder cumulants are close.

The Binder cumulant for the chiral condensate is used to probe the nature (as well as the position) of the transition. In the infinite volume limit, $B_{4}=3$ at a crossover, $B_{4}=1$ at a first-order transition and $B_{4}=1.604(1)$ at a 3 -dimensional Ising critical point. If there indeed were a critical endpoint, for $m>m_{c}(0) B_{4}$ would start at a value above the Ising value for $\mu_{I}=0$ (close to 3 for really large lattices) and decrease, passing through a value close to the Ising value at the critical endpoint, eventually approaching 1 for large $\mu_{I}$. On large enough lattices, finite-size scaling predicts that lines of $B_{4}$ versus $\mu_{I}$ for different lattice sizes will cross at the Ising value. Similarly lines of $B_{4}$ versus $m$ for different size lattices will cross at the Ising value as $m$ is varied.

In figure 1 we plot the Binder cumulants at the transition point as functions of $\mu_{I}^{2}$ for $m=0.025, m=0.03$ and $m=0.035$ for the various lattice sizes. For $m=0.035 B_{4}$ on the $12^{3} \times 4$ lattice starts at a value significantly above that for an Ising critical point and appears to increase with increasing $\mu_{I}$, and hence shows no evidence for a critical endpoint the slope of this straight line fit is $0.68(26)$. Similarly, for $m=0.03, B 4$ starts above the Ising value and increases with increasing $\mu_{I}^{2}$ on the $12^{3} \times 4$ and $16^{3} \times 4$ lattices. For the $12^{3} \times 4$ lattice the slope is $0.39(22)$, and for the $16^{3} \times 4$ lattice, this slope is $0.76(53)$. For $m=0.025$, there is no evidence for any $\mu_{I}^{2}$ dependence for $B_{4}$ on the $12^{3} \times 4$ lattice and 
it remains below the Ising value for the range of $\mu_{I}^{2}$ considered. We note that the $8^{3} \times 4$ slopes appear negative for $m=0.035$ and $m=0.025$ and positive for $m=0.03$, which we interpret as meaning that we have insufficient statistics to determine the very small slopes of the $8^{3} \times 4$ lines.
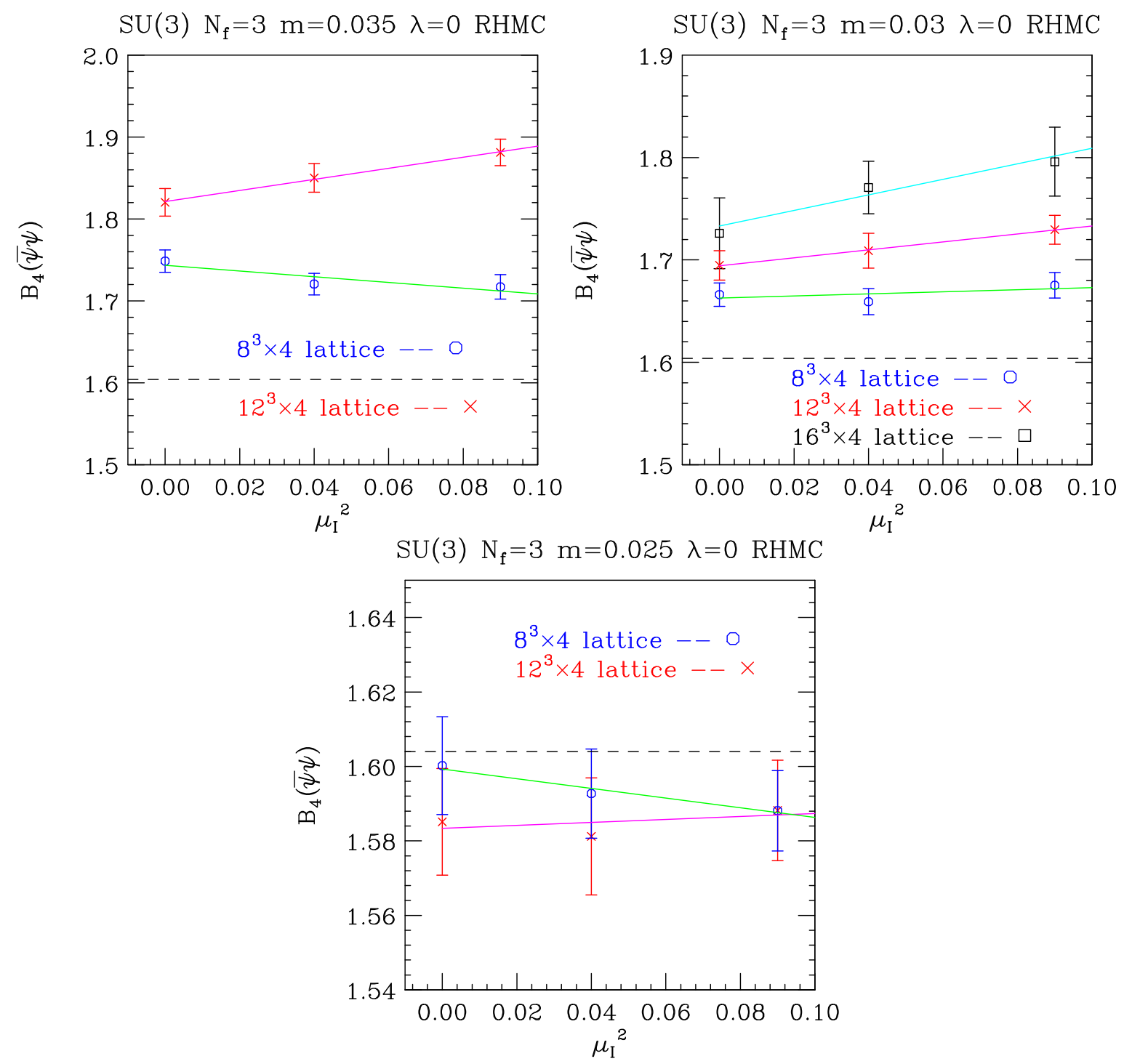

FIG. 1: Graphs showing the $\mu_{I}^{2}$ dependence of the Binder cumulants for the chiral condensate $\bar{\psi} \psi$ at the transition: a) for $m=0.035$, b) for $m=0.03$, c) for $m=0.025$. The dashed line is at the Ising value.

None of the slopes we have measured is much more than $2 \frac{1}{2}$ standard deviations from zero. However, the fact that the two $12^{3} \times 4$ slopes and the one $16^{3} \times 4$ slope for $m>m_{c}$ 
are all positive makes it less likely that this is a statistical fluctuation. For $m<m_{c}$ we can draw no conclusions.

We now turn our attention to the mass dependence of $B_{4}$ at fixed $\mu_{I}$ values. Figure 2 shows the $m$ dependence of $B_{4}$ for $\mu_{I}=0, \mu_{I}=0.2$ and $\mu_{I}=0.3$. First we note that
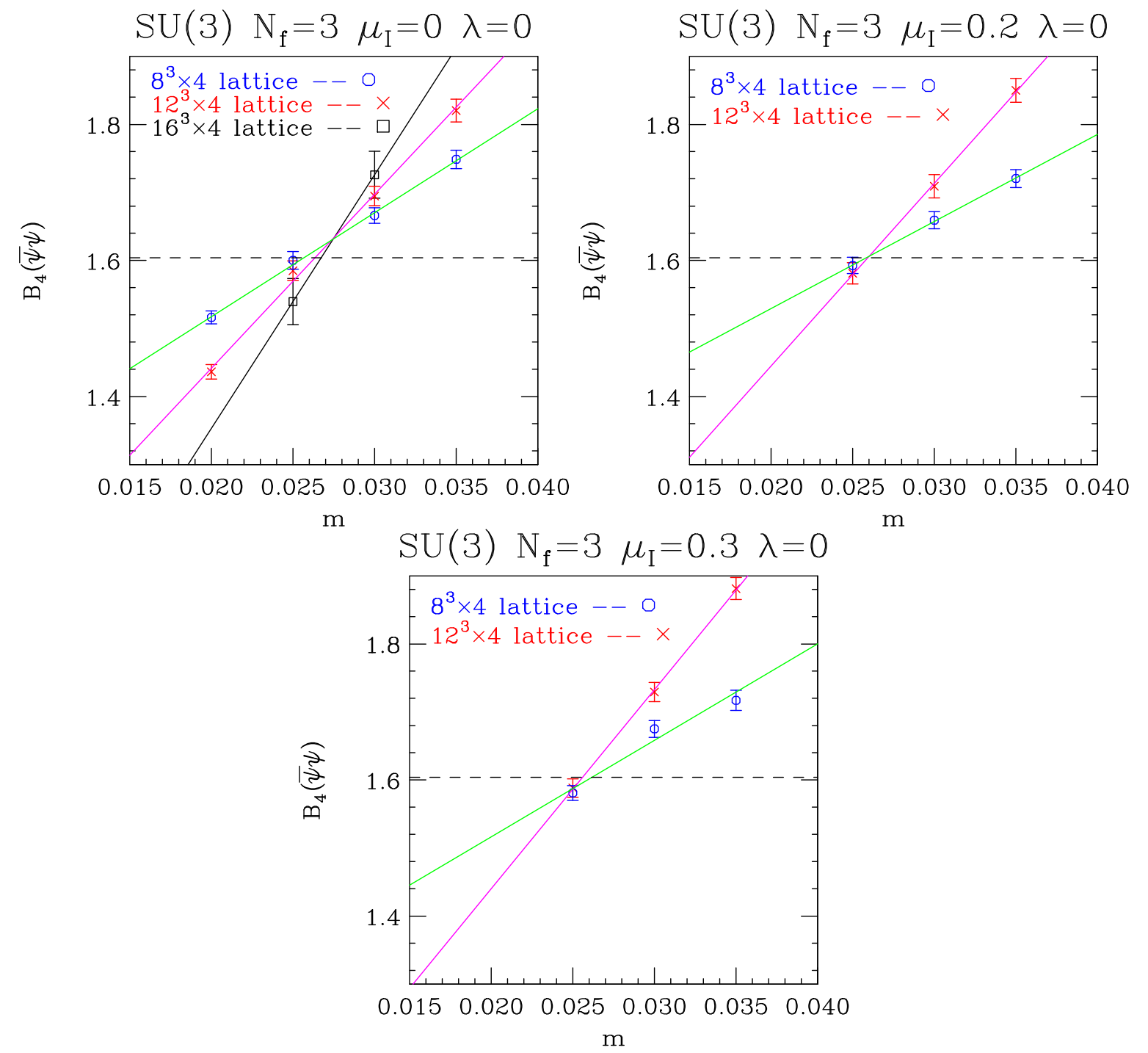

FIG. 2: Graphs showing the $m$ dependence of the Binder cumulants for the chiral condensate $\bar{\psi} \psi$ at the transition: a) for $\left.\mu_{I}=0, \mathrm{~b}\right)$ for $\left.\mu_{I}=0.2, \mathrm{c}\right)$ for $\mu_{I}=0.3$. The dashed line is at the Ising value.

the intersection of the curves for the different lattice sizes intersect at $B_{4}$ close to its value for the 3-dimensional Ising model. This is strong evidence that this critical point is in the universality class of the 3-dimensional Ising model, as predicted. We therefore use the 
masses for which the $12^{3} \times 4$ Binder cumulants achieve the Ising value as our estimate for the position of the critical point for the $\mu_{I}$ under consideration. We get $m_{c}(0)=0.0265(3)$, $m_{c}(0.2)=0.0259(5)$ and $m_{c}(0.3)=0.0256(4)$. A straight line fit yields

$$
m_{c}\left(\mu_{I}\right)=0.0265(3)-0.10(6) \mu_{I}^{2}
$$

This suggests that $m_{c}$ decreases with increasing $\mu_{I}$, rather than increasing as would be needed for a critical endpoint. Note also that if we were to use the intersections of the curves for different lattice sizes as our estimates for $m_{c}$, this would slightly increase our estimate for $m_{c}(0)$, slightly decrease our estimate of $m_{c}(0.3)$ and leave our estimate of $m_{c}(0.2)$ essentially unchanged. This would make the slope even more negative. In addition, since the transition temperature decreases with increasing $\mu_{I}, m_{c}$ in physical units will decrease slightly faster than the $m_{c}$ in lattice units, which we have presented here.

We have also examined the Binder cumulants for the isospin density $j_{0}^{3}$, and find that these are consistent with those for the corresponding chiral condensates. However, since our estimates for $j_{0}^{3}$ are much noisier, the errors on $B_{4}\left(j_{0}^{3}\right)$ are considerably larger than those for $B_{4}(\bar{\psi} \psi)$, which makes them less useful. The Binder cumulants for the plaquettes are appreciably larger, which is expected, since these should be a reasonable approximation to the energy-like order parameter whose Binder cumulant would approach 3 , even in the first-order regime and at the critical point.

The critical behaviour of this theory will be described by an effective Hamiltonian which is a linear combination of 3 fields, each of which has finite size scaling properties with one critical exponent. One such field has the scaling behaviour of a 'magnetization', a second that of an 'energy' and the third that of a 'density'. Each will be given as a linear combination of $\bar{\psi} \psi, S_{g}=\left(1-\frac{1}{3} \operatorname{Tr}_{\square} U U U U\right)$ and $j_{0}^{3}$. The simpler case at $\mu_{I}=0$, where there are only 2 fields to consider, has been studied in reference [1]. In that paper they were able to find simple expressions for the two 'mixing' parameters. In our case there are six such 'mixing' parameters, we have been unable to find the six equations required to determine these coefficients. If we were able to obtain these eigenmodes of the renormalization group, the Binder cumulant of the 'magnetization' order parameter would pass through the Ising value, once our lattice is large enough that subdominant terms in finite size scaling relation could be ignored. Until we can find such relationships we use the fact that, on large enough lattices, the magnetization component of the chiral condensate will dominate and its Binder 
cumulant will approach that of this eigenmode. The fact that the Binder cumulants for the chiral condensate cross close to the Ising value is evidence that this field is not strongly affected by contamination from non-magnetic eigenmodes, on the lattice sizes we use.

Using Ferrenberg-Swendsen reweighting again, we calculate the chiral susceptibilities and measure the positions and values of the peaks. We observe that the positions of these peaks are very close to the minima of the Binder cumulants. Finite size scaling tells us that, at the critical point,

$$
\chi_{\bar{\psi} \psi}\left(L, T_{c}\right)=L^{\frac{\gamma}{\nu}} \tilde{\chi}
$$

where $L$ is the spatial extent of the lattice and $T_{c}$ is the critical temperature. Hence if we plot $L^{-\frac{\gamma}{\nu}} \chi_{\bar{\psi} \psi}\left(L, T_{c}\right)$ as functions of $m$ for different $L$ values, the curves should cross at the critical point. In figure 3 we plot this quantity for $L=8$ and $L=12$, for each of our $\mu_{I}$ values. Here we have taken $\gamma=1.237$ and $\nu=0.630$ as the required critical indices for the 3-dimensional Ising model.

Because it is clear that the points on this graph do not fall on straight lines and the curves for different lattice sizes cross at rather shallow angles, a quantitative estimate for the position of the crossing would be difficult to obtain. What is clear is that the curves for the different lattice sizes cross somewhere between $m=0.25$ and $m=0.3$ for $\mu_{I}=0$ and $\mu_{I}=0.2$ and close to $m=0.25$ for $\mu_{I}=0.3$, which is consistent with our estimates of $m_{c}\left(\mu_{I}\right)$ from Binder cumulants.

As well as trying to determine the nature of the finite temperature transition as a function of $\mu_{I}$, and measuring observables and susceptibilities, the positions of the minima in the Binder cumulants, and the positions of the maxima in the various susceptibilities yield predictions for $\beta_{c}$ the transition $\beta$ values. The $\mu_{I}$ dependence of $\beta_{c}$ will ultimately yield the $\mu_{I}$ dependence of the transition temperature $T_{c}$. This not only requires that we know $T_{c}$ at $\mu_{I}=0$, which we can obtain from the numerous measurements by other groups, but it also requires that we know the renormalization group running of $\beta$ with lattice spacing $a$. On the coarse lattices we use, 2-loop perturbative running of the coupling constant which has been used earlier, is clearly suspect. Hence we present only the $\mu_{I}$ dependence of $\beta_{c}$ in this paper. Associated with our present simulations aimed at determining the equation-of-state for phased-quenched (lattice) QCD, we will measure the running of $\beta$ directly with the same action and masses as are used here, on zero temperature lattices. At that time we will be able to predict the $\mu_{I}$ dependence of $T_{c}$. 

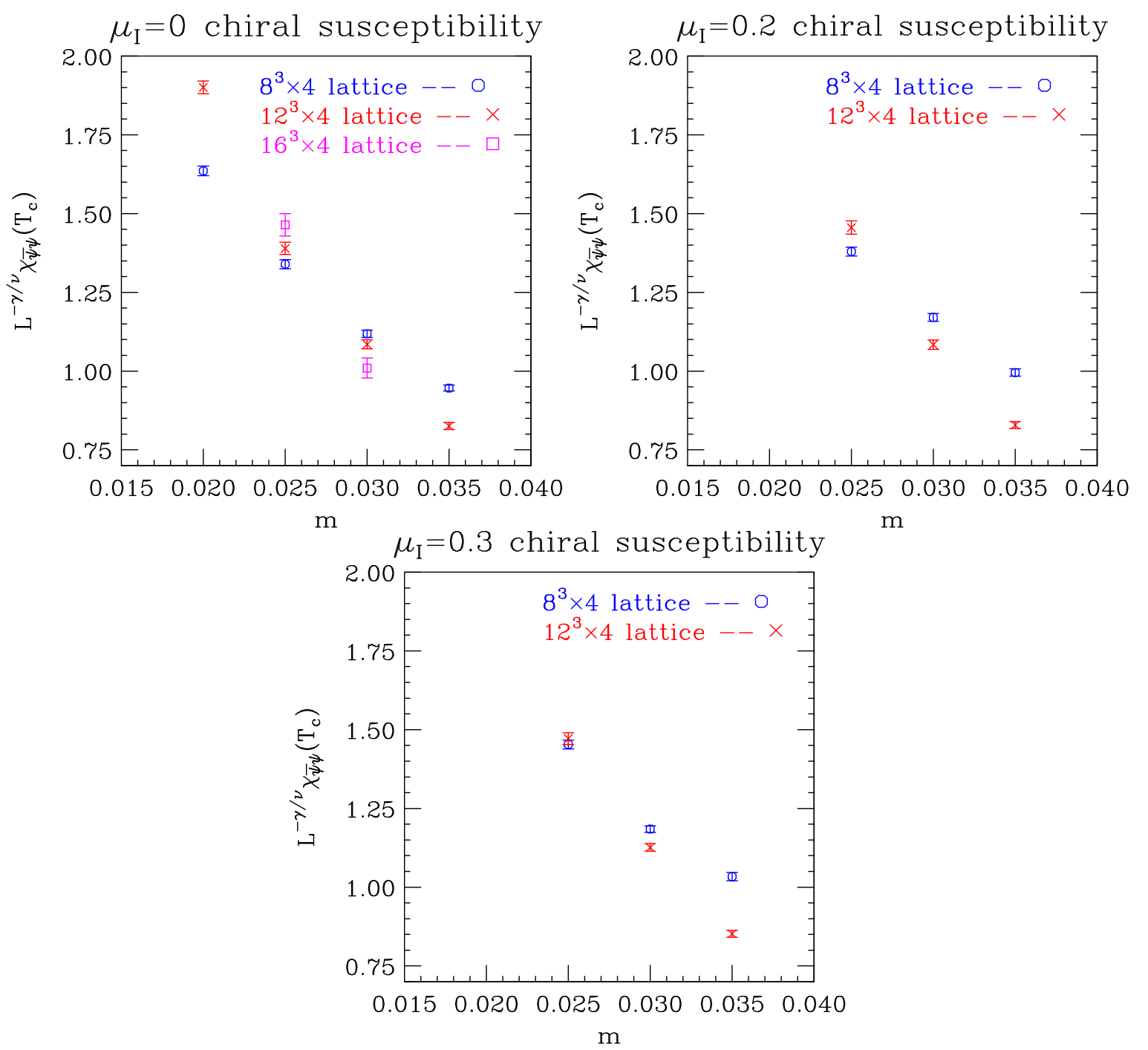

FIG. 3: The rescaled chiral susceptibilities $\chi_{\bar{\psi} \psi}$ as functions of $m$ : a) for $\mu_{I}=0, \mathrm{~b}$ ) for $\mu_{I}=0.2$, c) for $\mu_{I}=0.3$.

In figure 4 we plot the measured values of $\beta_{c}$ against $\mu_{I}^{2}$ for each of the quark masses. Straight line fits appear adequate with our current statistics. Although better fits could be obtained with a $\mu_{I}^{4}$ term for $m=0.025$ and $m=0.035$ - the $m=0.03$ straight line fit is excellent - the coefficients are clearly very small, and with only 3 points on each curve, such an exact fit is hard to justify. These fits are to the more extensive $12^{3} \times 4$ 'data'. We have plotted the $16^{3} \times 4$ points on the same graph. These indicate that the finite size effects on $\beta_{c}$ are very small. For comparison with the work of others, these fits are:

$$
\beta_{c}=5.13418(10)-0.1743(18) \mu_{I}^{2} \quad m=0.025
$$




$$
\begin{array}{ll}
\beta_{c}=5.14385(8)-0.1711(13) \mu_{I}^{2} & m=0.030 \\
\beta_{c}=5.15326(10)-0.1735(16) \mu_{I}^{2} & m=0.035
\end{array}
$$

and $\beta_{c}=5.12377(10)$ at $m=0.02, \mu_{I}=0$.

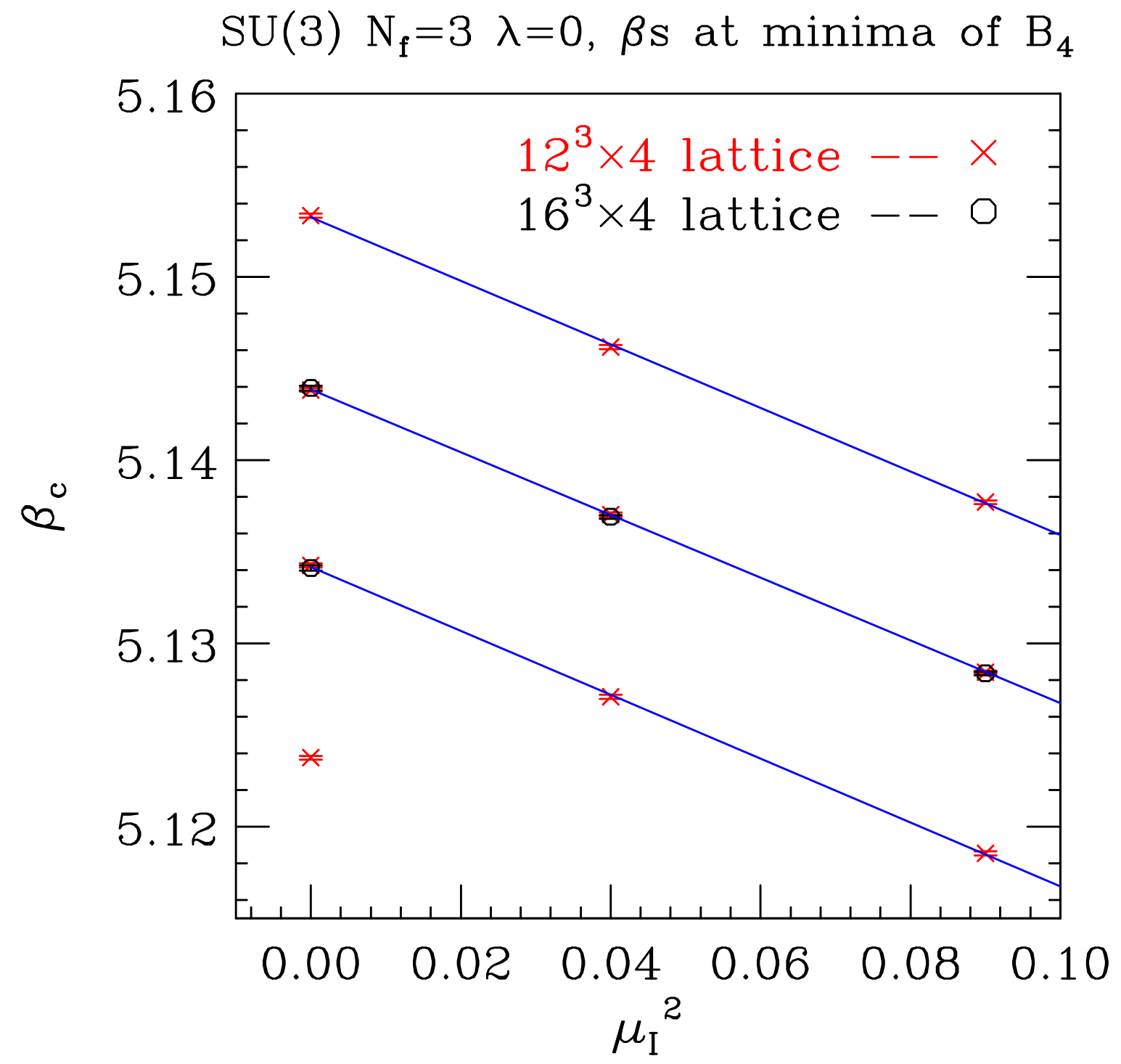

FIG. 4: Transition $\beta, \beta_{c}$ as functions of $\mu_{I}^{2}$ for chosen masses. The lines from top to bottom are for $m=0.035, m=0.03$ and $m=0.025$. The isolated point is for $m=0.02$.

\section{REWEIGHTING STUDIES}

As we saw in the previous section, the weak dependence of the Binder cumulants on $\mu_{I}$, and the sizable statistical errors in determining this fluctuation quantity mean that the 
observation that $B_{4}$ increases with $\mu_{I}$, while strongly suggested is not definitive. Similar difficulties arise for simulations at imaginary $\mu$. Here, de Forcrand, Kim and Philipsen have circumvented this difficulty by calculating $\partial B_{4} / \partial \mu^{2}$ directly [26]. They do this by calculating $B_{4}(\mu)$ and $B_{4}(\mu+\delta \mu)$ in the same simulation. This is achieved by including the ratio of determinants

$$
\rho=\operatorname{det}\left[\mathcal{M}(\mu+\delta \mu)^{N_{f} / 8}\right] / \operatorname{det}\left[\mathcal{M}(\mu)^{N_{f} / 8}\right]
$$

as a weight in the measurement of $\bar{\psi} \psi(\mu+\delta \mu)$ from the same ensemble at chemical potential $\mu$ as is used to measure $\bar{\psi} \psi(\mu)$, namely

$$
\langle\bar{\psi} \psi(\mu+\delta \mu)\rangle_{\mu+\delta \mu}=\frac{\langle\rho \bar{\psi} \psi(\mu+\delta \mu)\rangle_{\mu}}{\langle\rho\rangle_{\mu}},
$$

and similar expressions for higher powers of $\bar{\psi} \psi(\mu+\delta \mu)$.

Since exact calculation of such determinants is expensive, de Forcrand et al. used unbiased stochastic estimators for the ratio of determinants, in particular,

$$
\rho=\left\langle\exp \left[-\eta^{\dagger} \mathcal{M}(\mu)^{-N_{f} / 16} \mathcal{M}(\mu+\delta \mu)^{N_{f} / 16} \mathcal{M}(\mu+\delta \mu)^{N_{f} / 16} \mathcal{M}(\mu)^{-N_{f} / 16} \eta+\eta^{\dagger} \eta\right]\right\rangle_{\eta}
$$

where $\eta$ is Gaussian noise. The advantage of this method is that any finite number of noise vectors gives an unbiased estimator of the determinant. These authors reweighted from $\mu=0$ and performed a multistep reweighting to $\mu=0.1$

Whereas it appears that de Forcrand et al. limited themselves to $8^{3} \times 4$ lattices, we are investigating applying this to $12^{3} \times 4$ lattices, since the results of the previous section make it unclear whether the slope $\partial B_{4} / \partial \mu_{I}^{2}$ is the same for $8^{3} \times 4$ lattices as it is for larger lattices. We first investigated the possibility of reweighting from $\mu_{I}=0$ to $\mu_{I}=0.1$ in a single reweighting, but analysis of a few configurations quickly convinced us that although the overlap might be reasonable, the fluctuations were so large as to make it impossible to obtain a reasonable estimate of the determinant without use of far more noise vectors than is reasonable. We then went back to a reweighting from $\mu_{I}=0$ to $\mu_{I}=0.01$ as a single step process, and one that could be used as a basis for a multistep reweighting to an even larger $\mu_{I}$. For this trial run we used 1500 configurations at $m=0.03$, separated by 200 trajectories. For each configuration we used 200 noise vectors with $\delta \mu_{I}=0.01$ and the same set of noise vectors with $\delta \mu_{I}=-0.01$, making use of the fact that the determinant for a single configuration remains unchanged under $\mu_{I} \rightarrow-\mu_{I}$ to remove $\mathcal{O}\left(\delta \mu_{I}\right)$ fluctuations in our noisy estimator. We used 1000 noise vectors for our noisy estimators for $\bar{\psi} \psi(0)$ and 
the same set for $\bar{\psi} \psi(0.01)$ and $\bar{\psi} \psi(-0.01)$. This effectively removes the errors in using noisy estimators of the condensate from consideration. The resulting estimate of $\partial B_{4} / \partial \mu_{I}^{2}$ is $3.1 \pm 4.1$ compared with the estimate $0.38 \pm 0.22$ obtained in the previous section. This indicates that, as expected, we need to use a $\delta\left(\mu_{I}^{2}\right)$ much greater than the 0.0001 used here, which will require a multistep reweighting in order to avoid large fluctuations. Our estimate for $\partial \beta_{c} / \partial \mu_{I}^{2}$ is $-0.177(9)$, in agreement with $-0.171(1)$ obtained in the previous section. Figure 5 shows the noisy estimators of the determinants with errors that we obtained. We see that the errors are comparable with the difference of these determinant ratios from one and from their mean, which is one reason why the signal/noise ratio is so poor.

We notice with the reweighting from $\mu_{I}=0$, that one problem is that the signal is of order $\delta\left(\mu_{I}^{2}\right)=\left(\delta \mu_{I}\right)^{2}$, while the noise is of order $\delta \mu_{I}$. While this can be overcome with a multistep (multiple $\mu_{I} \mathrm{~s}$ ) reweighting, an alternative way of avoiding this difficulty is to start at nonzero $\mu_{I}$ where for small $\delta \mu_{I}, \delta \mu_{I}$ and $\delta\left(\mu_{I}^{2}\right)$ are of the same order of magnitude. We have thus tried 1-step reweighting from 1500 configurations at $\mu_{I}=0.2$, with $\delta \mu_{I}=0.01$ and hence with $\delta\left(\mu_{I}^{2}\right)=0.0041$. For this test we ran first with 200 noise vectors for each configuration, and later with 1000 noise vectors for each configuration. 1000 noise vectors were used in estimating the chiral condensate. Using 200 noise vectors to estimate the determinant ratio we obtained $\partial B_{4} / \partial \mu_{I}^{2}=-0.39 \pm 0.56$ and for 1000 noise vectors $-0.54 \pm 0.45$. Although this indicates that we still do not have enough statistics, we would only need to reduce the statistical errors by an order of magnitude to make a definitive prediction. $\partial \beta_{c} / \partial \mu_{I}^{2}$ measured in the same calculations is $-0.175(2)$ compared with $-0.171(1)$ calculated in the previous section. In figure 6, we show our estimates of the ratio of fermion determinants. Even with 200 noise vectors/configuration, the ratio of determinants is well determined. The statistical errors are considerably smaller than the ratio's departure from unity and, more importantly, considerably smaller than the range of values taken by this ratio over the ensemble of configurations. This presumably is why little improvement in the estimate of $\partial B_{4} / \partial \mu_{I}^{2}$ is obtained by increasing the number of noise vectors from 200 to 1000. Comparison of the estimates of the determinant ratios for 200 and 1000 noise vectors makes us confident that our noisy estimates are reliable.

In case the main problem was overlap, we reduced our $\delta \mu_{I}$ to 0.005 . The results, however, were similar. $\partial \beta_{c} / \partial \mu_{I}^{2}$ was well determined, while the errors in $\partial B_{4} / \partial \mu_{I}^{2}$ exceeded the signal. The determinant ratio was well determined by 200 noise vectors, the errors being much 

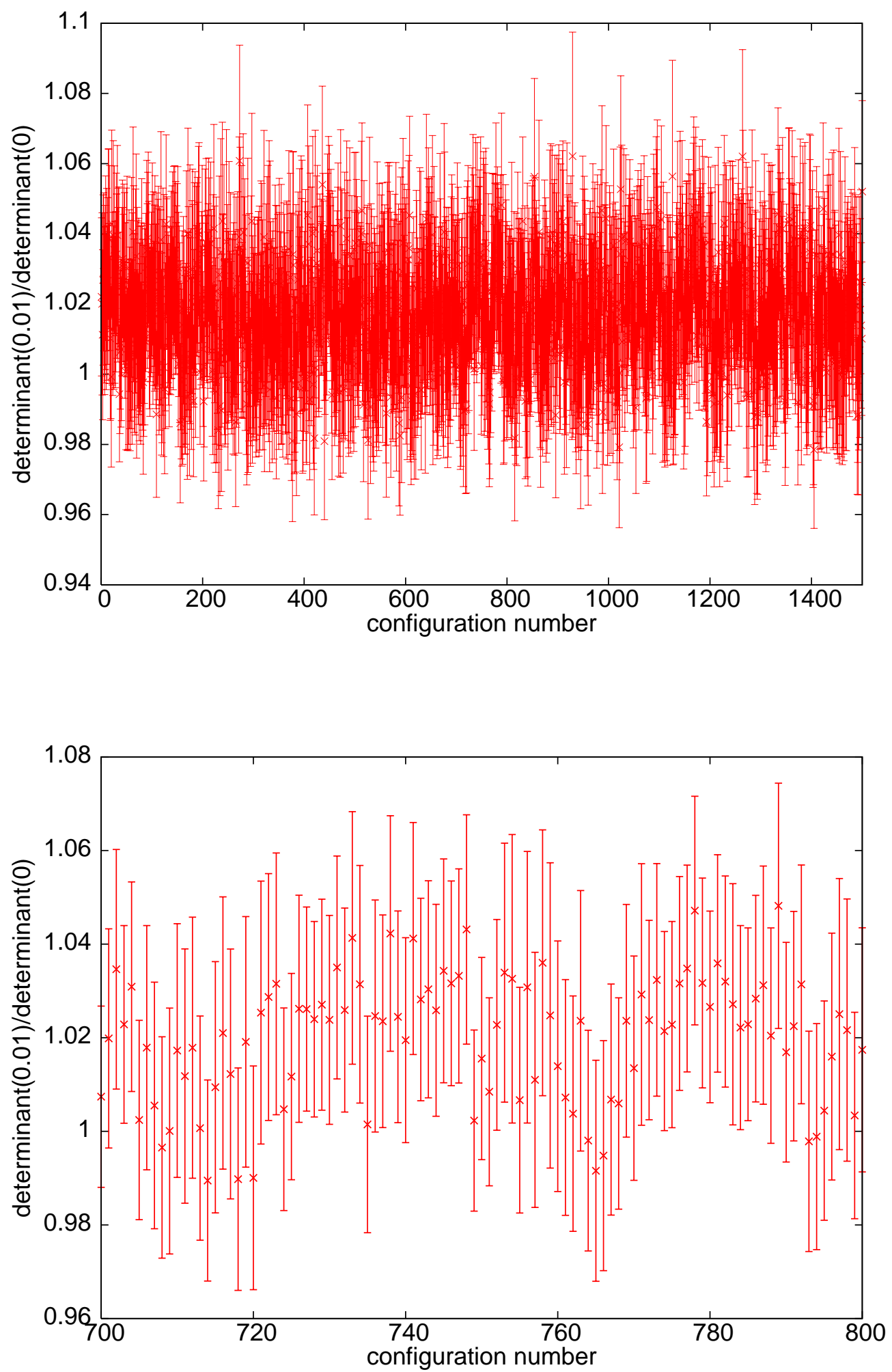

FIG. 5: a) Stochastic estimates of the ratio of fermion determinants at $\mu_{I}=0.01$ and $\mu_{I}=0$ on a $12^{3} \times 4$ lattice at $m=0.03, \mu_{I}=0, \beta=5.143$. b) Section of graph (a) showing detail. 

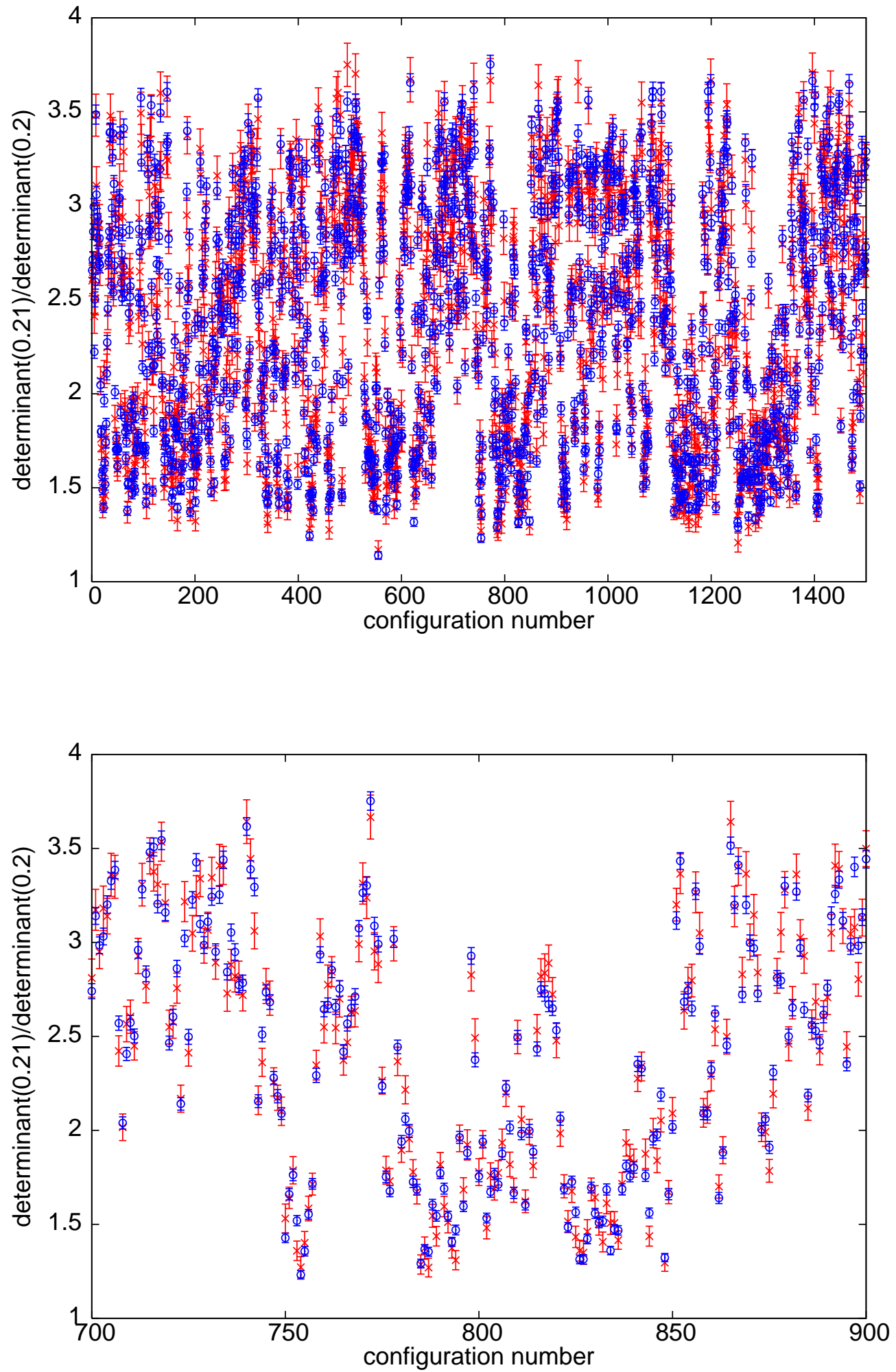

FIG. 6: a) Stochastic estimates of the ratio of fermion determinants at $\mu_{I}=0.21$ and $\mu_{I}=0.2$ on a $12^{3} \times 4$ lattice at $m=0.03, \mu_{I}=0.2, \beta=5.137$. The crosses(red online) are for 200 noise vectors; The circles(blue online) are for 1000 noise vectors. b) Section of graph (a) showing detail. 
smaller than the fluctuations in the value of this ratio from configuration to configuration.

To get an accurate estimate of $\partial B_{4} / \partial \mu_{I}^{2}$ will require the analysis of many more configurations. These configurations should be separated by enough trajectories as to make them reasonably independent or they are unlikely to improve the errors, just as increasing the number of noise vectors for estimating each ratio from 200 to 1000 did not significantly improve our errors. In fact, using 1 noise-vector to estimate the determinant ratio at the end of each of 300,000 consecutive trajectories at $\mu_{I}=0.2$, gave similar accuracy to using 200 or 1000 noise-vectors for each of our 1500 configurations spaced by 200 trajectories. In addition, producing a single trajectory takes much less computer time than a measurement with (say) a 100 noise-vector estimate of the determinant ratio and a 100 noise-vector estimate of $\bar{\psi} \psi$. In addition we need to check whether a single- or a multi-step estimation of the determinant ratio is more efficient, even at $\mu_{I}=0.2$ where it is not forced on us by other considerations.

\section{DISCUSSIONS AND CONCLUSIONS}

We have studied the finite temperature transition for 3-flavour lattice QCD with a finite chemical potential $\mu$ in the phase-quenched approximation, where the phase of the fermion determinant is set to zero, using RHMC simulations. This can be considered as studying lattice QCD with 3/2 up-type quarks and 3/2 down-type quarks at a chemical potential $\mu_{I}=2 \mu$ for isospin $\left(I_{3}\right)$. As we have indicated in the introduction, in the small $\mu\left(\mu_{I}\right)$ regime $-\mu_{I}<m_{\pi}$ - there are indications that the dependence of the transition temperature on $\mu$ for the phase-quenched model is similar if not identical to that for full QCD. Within the limitations of our statistics, which only allow us to include terms linear in $\mu^{2}$, we find that the coefficient of $\mu^{2}$ in the fit to $\beta_{c}\left(\mu^{2}\right)$, is within $10 \%$ and probably within $5 \%$ of that obtained by de Forcrand and Philipsen for the full theory by continuation from imaginary $\mu$ [2, 26]. This gives further evidence that the $\mu^{2}$ dependence of $T_{c}$ is the same in phase-quenched and full QCD. If this is true, it is reasonable to expect that the nature of the transition will be the same in both theories.

Let us briefly digress to discuss other recent work which could have relevance to the connection between phase-quenched and full QCD. Some recent work of Fodor, Katz and Schmidt, which employs the density-of-states method, uses the phase-quenched theory as a 
starting point for their factorized reweighting [29]. This shows a small but finite shift in $\beta_{c}$ in reweighting from phase-quenched to full QCD. However, all the $\mu$ values which they consider are larger than $m_{\pi} / 2$, and so in the region where the two theories are no longer expected to be similar. There has also been extensive work on QCD at finite chemical potentials using a random matrix/chiral perturbation theory approach [30, 31]. This has indicated that the phase of the fermion determinant becomes much worse behaved at for $\mu>m_{\pi} / 2$. Although this work does not (yet) explain why the full and phase-quenched QCD behave similarly, it does indicate that the same pion modes describe the physics of each, and suggests model calculations which might clarify the situation.

It was expected that the critical point at zero chemical potential would move to higher mass at finite chemical potential. If so, for quark masses just above the critical mass at $\mu=\mu_{I}=0$, this would become the sought-after critical endpoint where the crossover at $\mu=0$ would change to a first-order transition. Our simulations for $m$ close to $m_{c}(0)$ indicate that this does not happen, but rather $m_{c}\left(\mu_{I}\right)$ decreases with increasing $\mu_{I}$. The $\mu_{I}$ dependence of the Binder cumulant used to determine the nature of the transition is very weak for the lattice sizes we use $\left(8^{3} \times 4,12^{3} \times 4\right.$ and $\left.16^{3} \times 4\right)$. For this reason, our results can only be considered suggestive, and not definitive. Similar conclusions have been drawn by de Forcrand et al. from simulations at imaginary $\mu[2,26]$. This disagrees with the early work of the Bielefeld-Swansea collaboration [21], who did claim to find such a critical endpoint. However, as indicated before, these simulations used the $\mathrm{R}$ algorithm which as de Forcrand and Philipsen and we discovered can lead to misleading results.

De Forcrand et al. have recently introduced reweighting methods which enabled them to calculate the slope of the Binder cumulant directly, thus reducing the errors to a point where the sign is determined unambiguously [26]. This shows that the critical mass does indeed decrease with increasing $\mu$, so that there is no critical endpoint associated with $m_{c}(0)$. However, their published results using this new method are all on $8^{3} \times 4$ lattices where finite size effects, such as the fact that the chiral condensate is not the true order parameter (in the renormalization group sense), are large.

For this reason we have been investigating the use of such reweighting techniques for phase-quenched QCD on $12^{3} \times 4$ lattices. Larger lattices are less suited to such reweighting because the overlap between the ensembles of configurations at $\mu_{I}$ and $\mu_{I}+\delta \mu_{I}$ for given $\mu_{I}$ and $\delta \mu_{I}$ is smaller for larger lattices. The ratio of determinants is further from unity 
for the larger lattices, and the fluctuations associated with the noisy estimator on a single configuration are also larger. Correlations in molecular-dynamics time are longer on the larger lattice. Our tests are promising and suggest that using finite rather than zero $\mu_{I}$ configurations for the reweighting are preferable. However, since reweighting is expensive, unless we can find a way to make better use of the fact that the ratio of fractional powers of Dirac operators for $\mu_{I}$ and $\mu_{I}+\delta \mu_{I}$ is better conditioned than either of the original operators, reweighting will be considerably more expensive than the cost of producing a single trajectory, so that it is unclear as yet whether reweighting will prove to be the most cost-effective method of getting definitive results on these larger lattices.

One might ask whether our failure to find a critical endpoint disagrees with the work of Fodor and Katz [3]. They reported a critical endpoint at $\mu_{B}=360(40) \mathrm{MeV}$ and $T=$ 162(2) $\mathrm{MeV}$, and hence $\mu_{I}=240(27) \mathrm{MeV}$. Since our method breaks down for $\mu_{I} \gtrsim m_{\pi} \approx$ $140 \mathrm{MeV}$, their value is beyond the reach of our method. Hence our simulations do not show the absence of a critical endpoint, only the absence of a critical endpoint associated with the critical point at $\mu=\mu_{I}=0$, for 3-flavour QCD.

We are now extending our simulations of 3-flavour phase-quenched lattice QCD to enable a calculation of the equation-of-state of this theory outside the superfluid region. This will enable comparison with full QCD. In addition, the phase-diagram of QCD at finite isospin chemical potential and its equation-of-state are of interest in their own right. This has led to new activity in the studies of these theories [32].

All our simulations have been performed with the standard staggered action, with $N_{t}=4$ and are thus subject to large discretization errors. The Bielefeld-Swansea collaboration found that the critical mass at $\mu=0$ decreased dramatically, when they changed their lattice action from the standard lattice action to a highly improved action, indicating that this mass is very sensitive to finite lattice-spacing errors [21, 22]. A less dramatic decrease in the critical mass has recently been reported by de Forcrand, Kim and Philipsen when they increased $N_{t}$ from 4 to 6 with the standard staggered action [26]. Hence we should consider repeating our simulations at larger $N_{t}$, improving the action we use, or both.

In using a staggered action for 3 flavours, we are ignoring the so-called 'rooting' controversy. People have questioned whether taking fractional powers of the fermion determinant to allow use of staggered fermions to simulate numbers of fermion flavours which are not multiples of 4 , defines a theory with a sensible continuum limit. We direct the reader to 
[33, 34] for recent arguments on both sides of this controversy. Even assuming that this controversy is resolved and indicates that 'rooted' staggered fermions are legitimate, this is only relevant to the case of zero $\mu$. At $\mu \neq 0$, Golterman, Shamir and Svetitsky have pointed out that further ambiguities arise with regard to taking fractional powers of the phase of the determinant [35]. We have avoided this difficulty by ignoring the phase, but eventually it will need to be faced.

\section{Acknowledgements}

We thank $\mathrm{Ph}$. de Forcrand for his help and for useful discussions. We also thank O. Philipsen and F. Karsch for helpful discussions. The simulations reported here were performed on Jacquard and Bassi at NERSC on an ERCAP allocation and on Tungsten, Copper, Abe and Cobalt at NSCA and DataStar at SDSC under an NRAC grant.

[1] F. Karsch, E. Laermann and C. Schmidt, Phys. Lett. B 520, 41 (2001) arXiv:hep-lat/0107020.

[2] P. de Forcrand and O. Philipsen, JHEP 0701, 077 (2007) arXiv:hep-lat/0607017.

[3] Y. Aoki, G. Endrodi, Z. Fodor, S. D. Katz and K. K. Szabo, Nature 443, 675 (2006) arXiv:hep-lat/0611014.

[4] Z. Fodor and S. D. Katz, JHEP 0404, 050 (2004) arXiv:hep-lat/0402006.

[5] C. R. Allton et al., Phys. Rev. D 66, 074507 (2002) arXiv:hep-lat/0204010.

[6] R. V. Gavai and S. Gupta, Phys. Rev. D 68, 034506 (2003) arXiv:hep-lat/0303013.

[7] P. de Forcrand and O. Philipsen, Nucl. Phys. B 642, 290 (2002) arXiv:hep-lat/0205016.

[8] M. D'Elia and M. P. Lombardo, Phys. Rev. D 70, 074509 (2004) arXiv:hep-lat/0406012.

[9] V. Azcoiti, G. Di Carlo, A. Galante and V. Laliena, JHEP 0412, 010 (2004) arXiv:hep-lat/0409157.

[10] J. Engels, O. Kaczmarek, F. Karsch and E. Laermann, Nucl. Phys. B 558, 307 (1999) arXiv:hep-lat/9903030].

[11] P. de Forcrand and S. Kratochvila, Nucl. Phys. Proc. Suppl. 153, 62 (2006) arXiv:hep-lat/0602024. 
[12] A. Alexandru, M. Faber, I. Horvath and K. F. Liu, Phys. Rev. D 72, 114513 (2005) arXiv:hep-lat/0507020.

[13] D. T. Son and M. A. Stephanov, Phys. Rev. Lett. 86, 592 (2001) arXiv:hep-ph/0005225.

[14] S. Hands, J. B. Kogut, M. P. Lombardo and S. E. Morrison, Nucl. Phys. B 558, 327 (1999) arXiv:hep-lat/9902034.

[15] J. B. Kogut and D. K. Sinclair, Phys. Rev. D 66, 034505 (2002) arXiv:hep-lat/0202028.

[16] S. Ejiri, Phys. Rev. D 69, 094506 (2004) arXiv:hep-lat/0401012.

[17] J. B. Kogut and D. K. Sinclair, Phys. Rev. D 70, 094501 (2004) arXiv:hep-lat/0407027].

[18] B. Klein, D. Toublan and J. J. M. Verbaarschot, Phys. Rev. D 68, 014009 (2003) arXiv:hep-ph/0301143.

[19] D. Toublan and J. B. Kogut, Phys. Lett. B 564, 212 (2003) arXiv:hep-ph/0301183.

[20] A. Barducci, R. Casalbuoni, G. Pettini and L. Ravagli, Phys. Rev. D 72, 056002 (2005) arXiv:hep-ph/0508117.

[21] F. Karsch, C. R. Allton, S. Ejiri, S. J. Hands, O. Kaczmarek, E. Laermann and C. Schmidt, Nucl. Phys. Proc. Suppl. 129, 614 (2004) arXiv:hep-lat/0309116.

[22] C. Schmidt, arXiv:hep-lat/0408047.

[23] D. K. Sinclair and J. B. Kogut, PoS(LATTICE 2007) 225 (2007) arXiv:0709.2367 [hep-lat].

[24] M. A. Clark and A. D. Kennedy, Phys. Rev. D 75, 011502 (2007) arXiv:hep-lat/0610047.

[25] J. B. Kogut and D. K. Sinclair, Phys. Rev. D 74, 114505 (2006) arXiv:hep-lat/0608017.

[26] P. de Forcrand, S. Kim and O. Philipsen, PoS (LATTICE2007) 178 (2007) arXiv:0711.0262 [hep-lat].

[27] K. Binder, Z. Phys. B 43, 119 (1981).

[28] A. M. Ferrenberg and R. H. Swendsen, Phys. Rev. Lett. 61, 2635 (1988).

[29] Z. Fodor, S. D. Katz and C. Schmidt, JHEP 0703, 121 (2007) arXiv:hep-lat/0701022.

[30] K. Splittorff and J. J. M. Verbaarschot, Phys. Rev. D 75, 116003 (2007) arXiv:hep-lat/0702011.

[31] K. Splittorff and J. J. M. Verbaarschot, Phys. Rev. D 77, 014514 (2008) arXiv:0709.2218 [hep-lat]].

[32] Ph. de Forcrand, M. A. Stephanov and U. Wenger, arXiv:0711.0023 [hep-lat].

[33] M. Creutz, arXiv:0711.2640 [hep-ph].

[34] C. Bernard, M. Golterman and Y. Shamir, arXiv:0712.2560 [hep-lat]. 
[35] M. Golterman, Y. Shamir and B. Svetitsky, Phys. Rev. D 74, 071501 (2006) arXiv:hep-lat/0602026.

[36] We choose to call these momenta rather than fields, since we leave open the possibility of adding a function of $\psi$ to the action, which does not change the physics, but destroys the partial integrability of the equations-of-motion along a trajectory. This just means adding a familiar potential term, whereas adding terms of higher order in the momenta is somewhat less familiar. 